\title{
Duas novas espécies de Caballerotrema (Trematoda: Echinostomatidae) do pirarucu e do aruanà (Osteoglossidae), com uma redefiniçào do gênero e uma redescriçào de C. brasiliense Prudhoe, 1960.
}

\author{
Vernon E. Thatcher $\left[{ }^{*}\right]$
}

\section{Resumo}

O gênero Caballerotrema Prudhoe, 1960, é redefinido e C. brasiliense Prudhoe, 1960, do pirarucu, Arapaima gigas (Cuvier) é redescrito. Também são descritas C. arapaimense, n. sp. do pirarucu, e C. aruanense, n. sp., do aruanã, Osteoglossum bicirrhosum Vandelli. Ambos os hospedeiros são peixes da família Osteoglossidae e foram capturados próximo à cidade de Manaus, Amazonas, Brasil. O gênero Caballerotrema foi transferido da subfamília Singhiinae Yamaguti, 1958, para Himasthłinae Odhner, 1910. Uma chave para a separação das três espécies do gênero é apresentada.

\section{INTRODUÇÃO}

Echinostomatidae Poche, 1926, é uma das maiores famílias de trematódeos digenéticos e o estágio adulto infecta principalmente aves. Yamaguti (1971) citou 46 gêneros em aves, 12 em mamíferos e só 3 em répteis (todas em crocodilianos). Segundo a mesma referência, 12 espécies, representando 6 gêneros, já foram encontrados no homem em várias áreas do mundo.

Embora metacercárias de Echinostomatidae sejam achadas com freqüência encistadas nos peixes, os helmintólogos tinham dúvidas de que peixes serviriam de hospedeiros definitivos para os adultos destes vermes. Yamaguti (1958) citou apenas Singhia thapari (Singh, 1943) da Índia como o único representante da família com adultos em peixes. Mas tarde, Prudhoe (1960) descreveu, como gênero e espécie novos, Caballerotrema brasiliense do pirarucu, Arapaima gigas (Cuvier) do Brasil. Esta espécie foi a única da família citada de peixes brasileiros por Travassos et al. (1969) . Yamaguti (1971) reconheceu esta espécie co- mo um parasita verdadeiro de peixes, incluindo-a, com a espécie da Índia, na subfamília Singhiinae.

No presente estudo, 3 pirarucus foram necropsiados, e equinostómeos encontrados em número de 13,80 e 130 , respectivamente. Estas coleções continham a espécie de Prudhoe e mais uma nova espécie do gênero que está descrita aqui. Dois aruanãs foram examinadas também, e encontrados espécimes do mesmo gênero em número de 15 e 16. Este material representa mais uma nova espécie de Caballerotrema.

É de notar, que o pirarucu e o aruanã, Osteoglossum bicirrhosum Vandelli, são os únicos representantes da família Osteoglossidae. É apresentada aqui uma redefinição do gênero, porque a diagnose original não é muito completa, e deve ser ampliada para receber mais duas espécies. C. brasiliense é redescrita para correção de alguns erros na descrição de Prudhoe.

\section{MÉTODOS E MATERIAIS}

Os peixes foram capturados nas águas próximas à cidade de Manaus, Amazonas, Brasil, pelos funcionários da Divisão de Peixe e Pesca do Instituto Nacional de Pesquisas da Amazonia. Os métodos empregados na preparação dos vermes foram os mesmos citados anteriormente (Thatcher, 1978, 1979). As medidas são dadas com os limites inferior e superior, seguidas pela média entre parênteses. Todas as medidas são em milímetros, exceto o tamanho dos ovos e dos espinhos, que estão em micra.

(") - Instituto Nacional de Pesquisas da Amazônia, Manaus. 
SEÇÄo SISTEMÁtTICA

ECHINOSTOMATIDAE Poche, 1926.

Caballerotrema Prudhoe, 1960.

\section{DIAGNOSE GENÉRICA}

Echinostomatidae, Himasthlinae; com as características da família e da subfamília. Corpo alongado, cilíndrico; com disco peristômico largo e achatado. Disco guarnecido lateral e dorsalmente de uma fileira de espinhos; cada lobo ventral do disco com um grupo de quatro espinhos, dispostos aos pares, sendo o primeiro de cada lado o maior. Cutícula espinhosa. Ventosa oral subterminal, pequena; com uma extensão membranosa antero-dorsal e lateral. Esôfago longo; bifurcação dorsal ao acetábulo. Acetábulo grande; na região anterior do corpo. Bolsa do cirro volumosa; estendendo-se bem posteriormente ao acetábulo. Vesícula seminal externa presente. Gônadas centrais. Glândulas vitelínicas dorso-laterais; ảesde um ponto anterior ao ovário até as terminações dos cecos. Parasitas intestinais de peixes de água doce.

Espécie tipo: C. brasiliense Prudhoe, 1960.

\section{Caballerotrema brasiliense Prudhoe, 1960.}

Hospedeiro: Arapaima gigas (Cuvier), "pirarucu".

Habitat: Intestino.

Prevalência: $3 / 3$.

Intensidade: $13-130$ helmintos por hospedeiro .

Procedência: Lago Janauacá, Manaus, Amazonas, Brasil.

Lâminas: Instituto Nacional de Pesquisas da Amazônia (INPA) .

DIAGNOSE ESPECÍFICA (baseada em 10 exemplares) .

Com as características do gênero. Corpo com 4,6-11,3 (6,4) de comprimento e 0,51-0,74 $(0,61)$ de largura. Disco peristômico com 29 espinhos (27 menores e 2 maiores); disco mede $0,60-1,0 \quad(0,75)$ de largura; os espinhos grandes medem $26-47 \times 67-90(32 \times 80) \mu$; os espinhos pequenos com 14-35 x 34-60 $(24 \times$
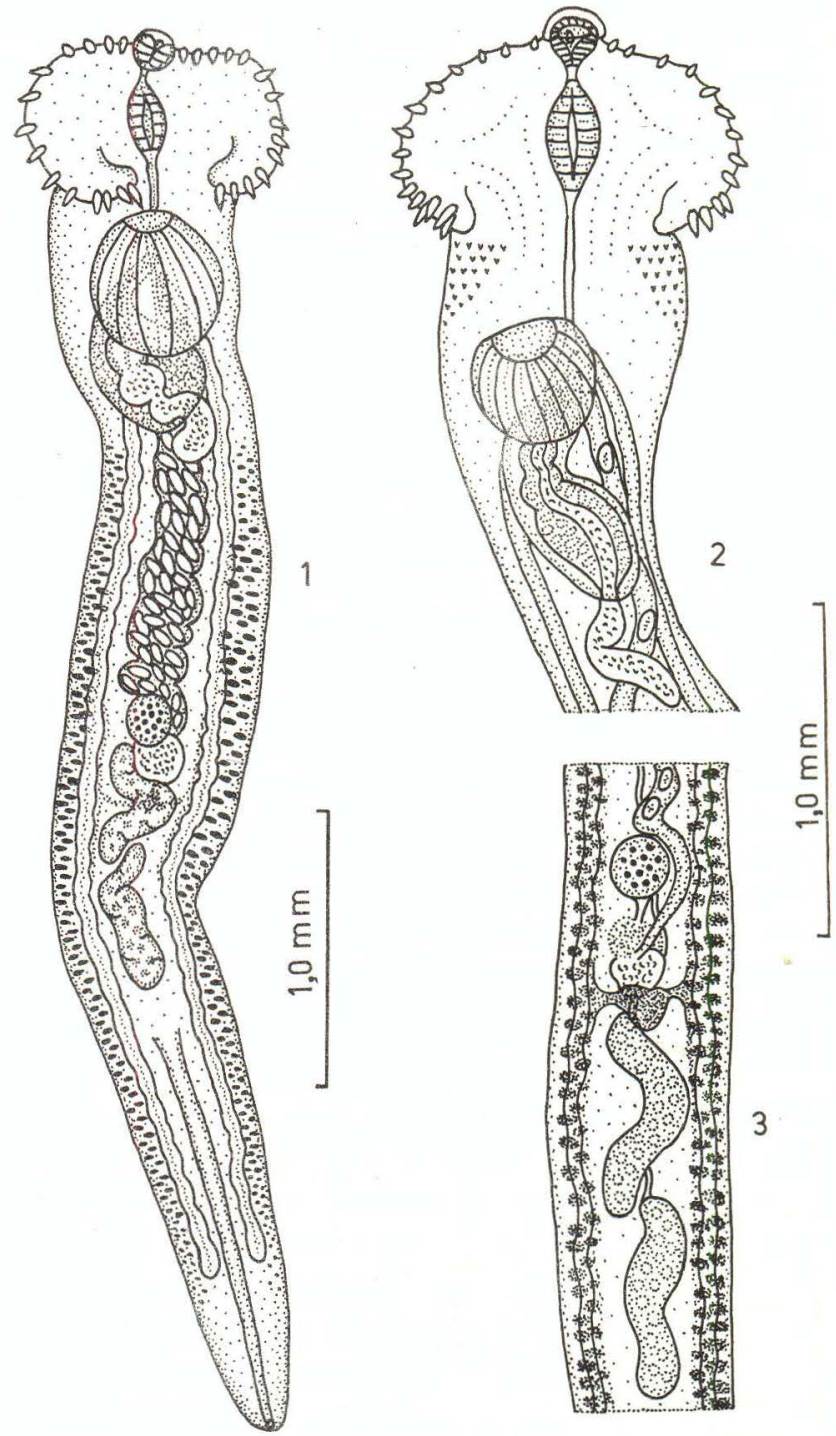

Est. 1: 1) - Caballerotrema arapaimense $\mathrm{n}$. $\mathrm{sp}$., visto ventral; 2) - C. brasiliense Prudhoe, 1960, visto ventral da extremidade anterior; 3) - C. brasiliense, visto ventral das gônadas.

50) $\mu$. Ventosa oral com $0,11-0,19(0,13)$ de diâmetro. Prefaringe curto. Esôfago longo com $0,25-0,45(0,33)$ de comprimento. Faringe com $0,19-0,30(0,22)$ de comprimento e 0,09-0,14 $(0,11)$ de diâmetro. Cecos estreitos, com $0,022-0,038(0,028)$ de largura. Acetábulo com 0,29-0,41 (0,32) de comprimento e 0,25-0,40 $(0,30)$ de largura. Testículos um pouco sinuosos. Testículo anterior com 0,38-0,63 $(0,45)$ de comprimento e 0,12-0,26 $(0,18)$ de largura. Testículc posterior com $0,37-0,70(0,50)$ de comprimento e 0,14-0,22 $(0,17)$ de largura. Bolsa do cirro com $0,38-0,64(0,52)$ de comprimento 
e 0,26-0,35 (0,31) de largura. Ovário esférico, com 0,14-0,21 $(0,16)$ de diâmetro. Cvos com $48-56 \times 80-84(51 \times 82) \mu$.

Caballerotrema arapaimense, n. sp.

Hospedeiro: Arapaima gigas (Cuvier), "pirarucu".

Habitat: Intestino.

Prevalência: $2 / 3$.

Intensidade: 1-19 helmintos por hospedeiro.

Procedência: Lago Janauacá, Manaus, Amazonas, Brasil.

Holótipo: Instituto Nacional de Pesquisas da Amazônia (INPA).

Parátipos: INPA e Museu de Zoologia da Universidade de São Paulo.

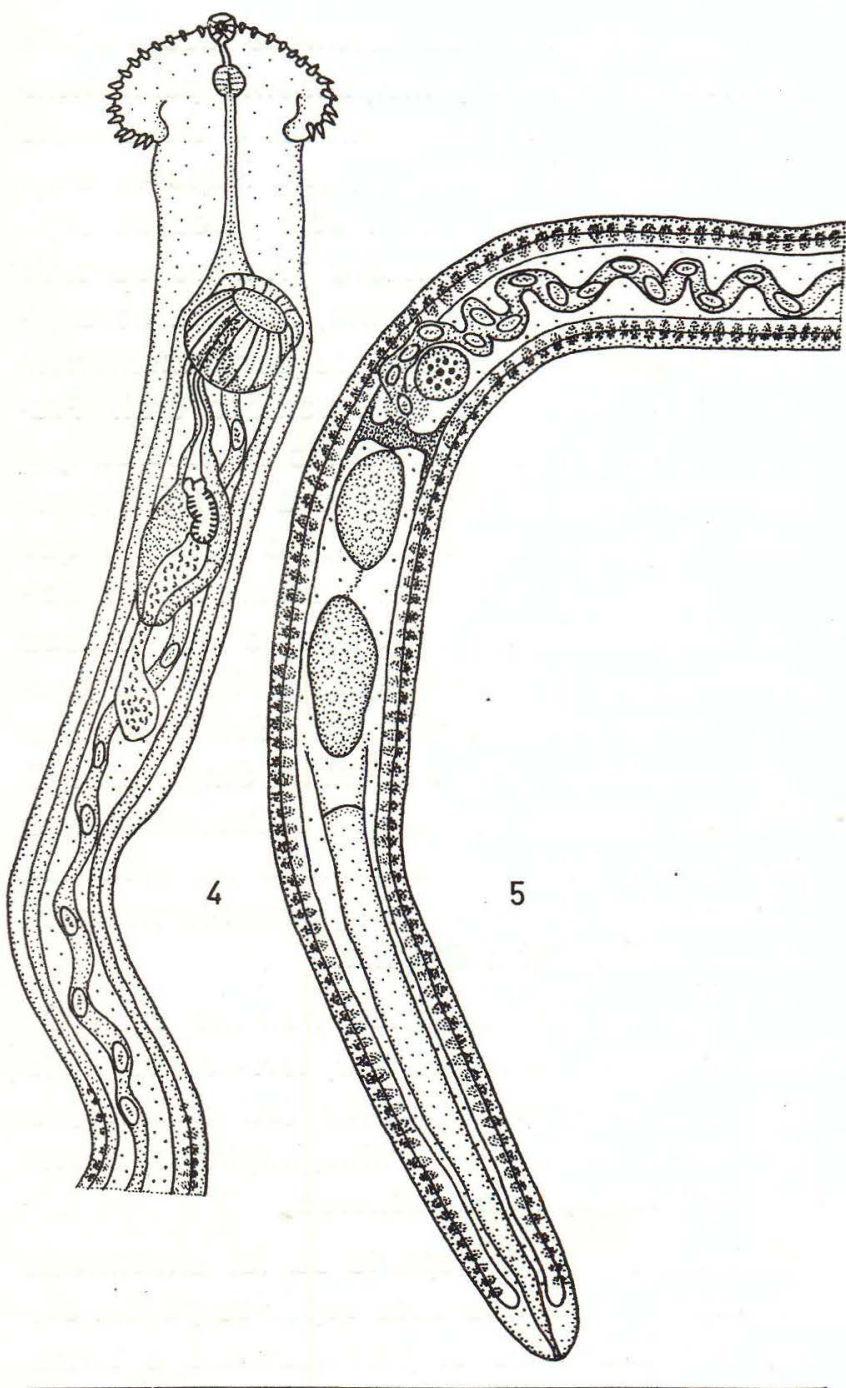

Est. II: 4 e 5) - C. aruanense $\mathrm{n}$. sp. visto ventral.
DIAGNOSE ESPECÍFICA (baseada em 10 exemplares).

Com as características do gênero. Corpo medindo 3,8-8,3 $\quad(5,4)$ de comprimento e $0,62-0,77(0,68)$ de largura. Disco peristômico com 29 espinhos (27 menores e 2 maiores); disco com 0,83-1,02 $(0,92)$ de largura; espinhos grandes com $27-48 \times 84-200(34 \times 130) \mu$; espinhos pequenos com 18-36 x 67-139 (24 x 80) $\mu$. Ventosa oral com $0,14-0,30(0,18)$ de diâmetro. Prefaringe curto. Esôfago longo com 0,31-0,36 $(0,33)$ de comprimento. Faringe com $0,26-0,36$ $(0,30)$ de comprimento e $0,11-0,27 \quad(0,17)$ de largura. Cecos estreitos, com 0,027-0,072 $(0,045)$ de largura. Acetábulo com 0,33-0,87 $(0,50)$ de comprimento e 0,29-0,56 $(0,38)$ de largura. Testículos sinuosos e indentados. Testículo anterior com 0,30-0,97 $(0,52)$ de comprimento e 0,19-0,30 $(0,16)$ de largura. Testículo posterior com $0,34-1,12(0,60)$ de comprimento e $0,11-0,32(0,17)$ de largura. Bolsa do cirro com $0,47-0,82(0,59)$ de comprimento por 0,22-0,42 $(0,24)$ de largura. Ovário esférico, com $0,15-0,24(0,17)$ de diâmetro. Ovos com $28-58 \times 72-83(48 \times 75) \mu$.

\section{Caballerotrema aruanense $n . s p$.}

Hospedeiro: Osteoglossum bicirrhosum Vandelli, "aruanã".

Habitat: Intestino.

Prevalência: $2 / 2$.

Intensidade: $15-16$ helmintos por hospedeiro.

Procedência: Lago Janauacá, Manaus, Amazonas, Brasil.

Holótipo: Instituto Nacional de Pesquisas da Amazônia (INPA).

Parátipos: INPA e Museu de Zoologia da Universidade de São Paulo.

DIAGNOSE ESPECÍFICA (baseada em 10 exemplares).

Com as características do gênero. Corpo medindo 5,8-9,1 $(7,8)$ de comprimento e 0,39-0,62 $(0,51)$ de largura. Disco peristômico com 29 espinhos (27 menores e 2 maiores); disco com $0,63-0,88(0,74)$ de largura; espinhos

Duas... 

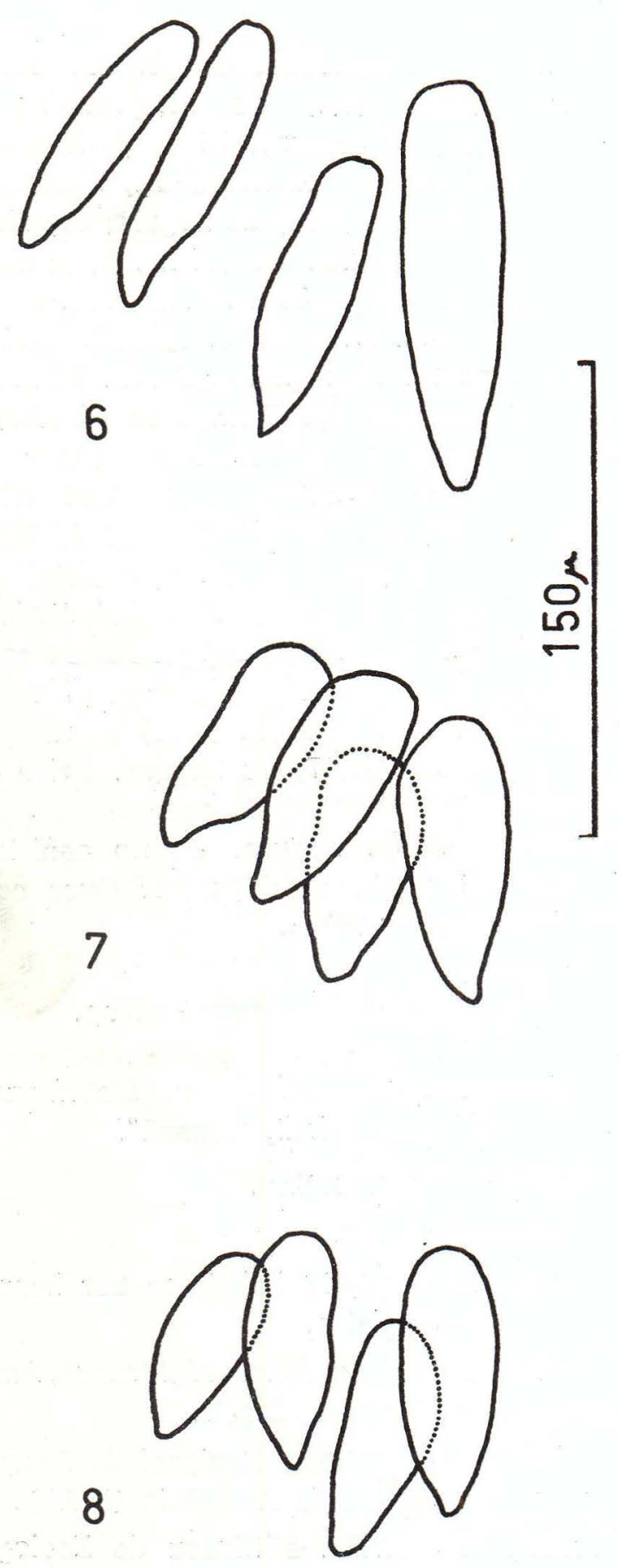

Est. III: 6) - Espinhos ventrais do disco peristômico de C. arapaimense; 7) - Espinhos ventrais do disco de C. brasiliense; 8) - Espinhos ventrais do disco de C. aruanense.

grandes com $24-32 \times 72-98(28 \times 88) \mu$; espinhos pequenos com 18-28 x 47-65 (24 x 53) $\mu$. Ventosa oral com $0,11-0,16(0,13)$ de diâmetro.
Prefaringe curto. Esôfago longo com 0,30-0,42 $(0,37)$ de comprimento. Faringe com $0,18-0,22$ $(0,20)$ de comprimento e 0,12-0,16 $(0,14)$ de diâmetro. Cecos estreitos, com 0,031-0,048 $(0,039)$ de largura. Acetábulo com 0,36-0,45 $(0,41)$ de comprimento e 0,31-0,42 $(0,36)$ de largura. Testículos arredondados ou ovalados. Testículo anterior com 0,30-0,54 $(0,44)$ de comprimento e $0,19-0,30(0,24)$ de largura. Testículo posterior com $0,36-0,66(0,53)$ de comprimento e 0,18-0,29 $(0,24)$ de largura. Bolsa do cirro com 0,74-1,2 $(0,94)$ de comprimento e $0,21-0,25(0,23)$ de largura. Ovário esférico, com $0,18-0,25(0,20)$ de diâmetro. Ovos com $43-60 \times 70-97(49 \times 79) \mu$.

\section{DISCUSSÃo}

Caballerotrema se distingue dos demais gêneros da família principalmente pela forma e tamanho do disco peristômico. O disco deste gênero, além de ser maior, tem lobos ventrais bem desenvolvidos e com dois pares de espinhos de cada lado, sendo o primeiro de cada grupo o maior. Dorsalmente, a fileira de espinhos é contínua, e uma pequena membrana projeta-se anteriormente à ventosa oral. Prudhoe (1960) tinha razão quando considerou que Caballerotrema se assemelha a Himasthla, parasita de aves. Infelizmente, o desenho que ele publicou foi feito com base em um espécime excessivamente contraído, o que causou confusão quanto à morfologia. Por isto, Yamaguti (1971) se enganou ao colocar Caballerotrema junto com Singhia na subfamília Singhiinae. O presente trabalho indica que o Caballerotrema se encaixa bem na subfamília Himasthlinae Odhner, 1910, e que morfologicamente tem pouco em comum com Singhia.

Entre as três espécies tratadas aqui, $C$. aruanense se distingue por ter: corpo mais fino; testículos arredondados (ou ovalados) e separados; disco peristômico, espinhos, acetábulo e faringe, todos menores.

Até agora esta espécie só foi encontrada no aruanã. As outras duas espécies podem ser diferenciadas entre si pelo tamanho e forma dos espinhos do disco peristômico e pela forma dos testículos. 
As três espécies de Caballerotrema podem ser separados com a ajuda da seguinte chave :

I. Testículos arredondados, ou ovalados: separados ......... C. aruanense.

II. Testículos alongados; dobrados ou sinuosos; contíguos .............

A. Espinhos pequenos do disco perịstômico com mais de $65 \mu$ de comprimento (o primeiro em média com 130). ........ C. arapaimense.

B. Espinhos pequenos do disco com menos de $62 \mu$ de comprimento (primeiro em média com 89) ........... C. brasiliense.

\section{SUMMMARY}

The genus Caballerotrema Prudhoe, 1960, was redefined and C. brasiliense Prudhoe, 1960, from the "pirarucú", Arapaima gigas (Cuvier), was redescribed. C. arapaimense n. sp. from the "pirarucu" and C. aruanense n. sp. from the "aruanã", Osteoglossum bicirrhosum Vandeli, were described. Both hosts are fishes of the family Osteoglossidae collected near Manaus, Amazonas, Brazil. Caballerotrema was transferred from the subfamily Singhiinae Yamaguti, 1958, and placed in $\mathrm{Hi}$ masthlinae Odhner, 1910. A key to the three known species of the genus was presented.

\section{BIBLIOGRAFIA}

PRUDHOE, S.

1960 - On two new parasitic worms from Brazil. Libro. Hom. Eduardo Caballero y Caballero. Univ. Nac. Auton. México. p. 415-418.

THATCHER, V.E.

1978 - Quatro espécies novas de Haploporidae (Trematoda: Digenea) de peixes de água doce de Colômbia com uma revisão do gênero Saccocoelioides Szidat, 1954. Acta Amazonica, 8 (3): 477-484.

1979 - Paramphistomidae (Trematoda: Digenea) de peixes de água doce: dois novos gêneros de Colômbia e uma redescrição de Dadaytrema oxycephala (Diesing, 1836) Travassos, 1934, da Amazônia. Acta Amazonica, 9 (1): 203-208.

Travassos, L.; Teixeira de Freitas, J.F. \& Kohn, A. 1969 - Trematódeos do Brasil. Mem. Inst. Oswal. do Cruz, 67: 1-886.

YAMAGUtT, S.

1958 - Systema Helminthum. Vol. I., Pts. 1 \& 2, The Digenetic Trematodes of Vertebrates. Intersci. Pub. Co. 1575p.

1971 - Synopsis of Digenetic Trematodes of Vertebrates. Keigaku Publ. Co., Tokyo. 1772p.

(Aceito para publicação em 02/01/80) 